\title{
Paeoniflorin inhibits the growth of bladder carcinoma via deactivation of STAT3
}

\author{
JIANHUI-YANG \\ YU REN \\ ZHONG-GUAN LOU \\ XUE WAN \\ GUO-BIN WENG* \\ DONG CEN* \\ Laboratory of Kidney Carcinoma \\ Ningbo Urology and Nephrology \\ Hospital, Urology and Nephrology \\ Institute of Ningbo University, Ningbo \\ Zhejiang 315000, P.R. China
}

Accepted December 20, 2017

Published online March 16, 2018

\begin{abstract}
Bladder cancer $(\mathrm{BCa})$ is one of the most common urinary cancers. The present study aims to investigate whether Paeoniflorin (Pae) can exert inhibitory effects on BCa. The results showed that Pae inhibited proliferation of human $\mathrm{BCa}$ cell lines in a concentration- and time-dependent manner. Pae and cisplatin (Cis) synergistically inhibited the growth of tumours in RT4-bearing mice. Pae treatment neutralized the body loss induced by Cis. Moreover, Pae induced apoptosis in RT4 cells and increased the activities of caspase3, caspase 8 and caspase 9 . Western blotting and immunohistochemical analysis revealed that the phosphorylated signal transducer and activator of transcription-3 (p-STAT3) level were decreased in Pae-treated RT4 cells and Pae-treated tumour-bearing mice. Furthermore, STAT3 transcriptional target B-cell lymphoma-2 was decreased in Pae-treated RT4 cells. Interestingly, Pae prevented translocation of STAT3 to the nucleus in RT4 cells. Collectively, Pae inhibits the growth of BCa, at least in part, via a STAT3 pathway.
\end{abstract}

Keywords: bladder cancer, Radix Paeoniae alba, paeoniflorin, apoptosis, STAT3

Bladder cancer $(\mathrm{BCa})$ is one of the most common malignancies in the western world. In the United States, it was estimated that there were 76,960 new cases and 16,390 deaths in 2016 (1). It was previously reported that approximately $30 \%$ of BCa patients had muscle and invasive cancer cells and $10 \%$ of patients suffered from metastatic disease, which resulted in a poor prognosis (2). Current application of chemotherapy, radiotherapy and radiochemotherapy has made great progress in preventing $\mathrm{BCa}$, and the overall survival has been improved. However, recurrence and metastasis of BCa occurs frequently, leading to unsatisfactory survival rates. Moreover, poor prognosis has persisted due to fatigue, pain and side effects induced by chemotherapy (3). Thus, there is a demand to develop a safe and effective drug for improving treatment and prognosis of BCa patients.

Signal transducer and activator of transcription-3 (STAT3), one of the STAT family members, is a downstream effector of various cytokines, growth factors and hormones (4). Fol-

\footnotetext{
*Correspondence; e-mail: cengdong2002@163.com, wgbningbo@126.com
} 
lowing activation, phosphorylated STAT3 (p-STAT3) dimerizes and translocates to the nucleus, where it finally regulates the expression of numerous critical genes that control cell proliferation and survival (5-6). A number of studies have been performed on BCa, which have highlighted STAT3 as a potential therapeutic target. In clinical specimens, it has been confirmed that urothelial BCa displays elevated expression as well as activation of STAT3 (7). Silencing of STAT3 suppresses the proliferation of T24 cells in vitro and in vivo compared to the controls, and overexpression of STAT3 prevents cells from undergoing morphological changes and apoptosis triggered by the Ha-ras oncogene (8-9). Moreover, inhibition of STAT3 by small interfering RNA or a specific inhibitor restores chemosensitivity in T24 cells (10), suggesting that STAT3 could be a major target for increasing chemosensitivity in patients.

Interest in the identification of novel anticancer agents derived from natural sources has been growing exponentially. Paeoniflorin (Pae) is the main active ingredient of Radix Paeoniae alba (RPA), which is used in traditional Chinese medicine to treat a range of inflammatory diseases (11). Today, there is interest in studying the therapeutic effects of Pae on different cancer types. In previous pharmacological investigations, Pae was confirmed to inhibit the growth of human pancreatic cancer, gastric carcinoma cells, colorectal carcinoma cells, and glioma cells (12-15). A new study (16) recently reported the anti-proliferative properties of RPA extraction in treating $\mathrm{BCa}$, both in vitro and in vivo. However, it is unknown whether Pae could inhibit BCa. Therefore, the main purpose of the present study was to estimate the anti-tumour activity of Pae in BCa.

\section{EXPERIMENTAL}

\section{Cell lines and culture}

Human BCa cell lines (RT4, 253J and J82) were originally obtained from the American Type Culture Collection (Manassas, VA, USA). The cells were maintained in Dulbecco's Modified Eagle Medium (Gibco-BRL, USA), supplemented with $10 \%$ foetal bovine serum (Sigma-Aldrich, USA), $4 \mathrm{mmol} \mathrm{L}^{-1}$ glutamine and $100 \mathrm{U} \mathrm{mL}^{-1}$ penicillin, at $37^{\circ} \mathrm{C}$ in $5 \% \mathrm{CO}_{2}$.

\section{3-(4,5-Dimethyl-2-thiazolyl)-2,5-diphenyl-2H-tetrazolium bromide (MTT) assay}

A total of $2 \times 10^{3}$ cells were seeded in a 96-well plate and then incubated with different concentrations of Pae (purity $>98 \%$; Chengdu Biopurify Phytochemicals Ltd., China) for 24-72 h. The cells were treated with $20 \mu \mathrm{L}$ of MTT (5 g L ${ }^{-1}$, Sigma-Aldrich) solution and incubated at $37^{\circ} \mathrm{C}$ for $4 \mathrm{~h}$. After discarding the medium, absorbance was recorded by a microplate reader (Biotek, Vermont, USA). Each cell viability assay was performed in triplicate. The relative cell viability (\%) was determined by comparing the absorbance with the control, which was treated with a phosphate buffered solution (PBS). The $I C_{50}$ value was determined according to the growth curve of the cells using the GraphPad Prism 5.0 software.

\section{Apoptosis assay}

Apoptosis in RT4 cells was detected using a cell apoptosis detection kit (BD Pharmingen, San Diego, CA, USA). Briefly, the cells were seeded $2.5 \times 10^{5}$ per well in 6 -well plates for $24 \mathrm{~h}$ and were then treated with Pae $(25$ or $50 \mu \mathrm{M})$ for $48 \mathrm{~h}$. The cells were labelled by 
Annexin V-fluorescein isothiocyanate (FITC) and stained using a binding buffer for $30 \mathrm{~min}$ in the dark according to the instructions. Subsequently, $10 \mu \mathrm{L}$ of propidium iodide (PI) was added to the cells and incubated for $15 \mathrm{~min}$ at room temperature in the dark. The apoptotic rate was measured by flow cytometry (FACS Calibur, BD Biosciences).

\section{Caspase activity assay}

Activities of caspase-3, caspase- 8 and caspase-9 in Pae-treated RT4 cells (48 h) were detected by caspase activity assay kits from Beyotime Biotechnology (China).

\section{Western blotting}

RT4 cells were pre-treated with Pae $(25$ or $50 \mu \mathrm{M})$ in a serum-free medium for $48 \mathrm{~h}$. Nuclear protein was extracted using a nuclear and cytoplasmic protein extraction kit provided by Beyotime Biotechnology (Nantong, China). The amount of protein was quantified by the bicinchoninic acid assay (Thermo Fisher Scientific, USA). Equal amounts of extract were separated by sodium dodecyl sulfate polyacrylamide gel electrophoresis and transferred to polyvinylidene difluoride membranes (Gibco-Life Technologies, USA). The membranes were incubated with a primary antibody and then probed with a secondary antibody and visualized by enhanced chemiluminescence. All western blot experiments were performed at least three times. The primary antibodies used were as follows: anti-B-cell lymphoma-2 (Bcl-2), anti-Bcl-2 associated X protein (Bax) and anti-p-STAT3, all purchased from Santa Cruz Biotechnology (USA). $\beta$-actin and histone were used as internal controls for relative quantification.

\section{In vivo tumour xenograft study}

Male BALB/c nude mice (20-22 g) were from the Shanghai Laboratory Animal Center Co., Ltd. (China). The animals were housed in temperature-controlled rooms $\left(22 \pm 2{ }^{\circ} \mathrm{C}\right)$, with relative humidity of $55 \pm 5 \%$ and a $12 \mathrm{~h}$ light/dark cycle. All protocols involved in animal experiments were approved by the Animal Care and Use Committee of Ningbo Urology and Nephrology Hospital (Qianhe road, No. 1, Yinzhou, Ningbo, China). Moreover, the animal experiments were also conducted according to the U.K. Animals (Scientific Procedures) Act, of 1986 and associated guidelines. RT4 cells $\left(5 \times 10^{6}\right)$ in $100 \mu \mathrm{L}$ of PBS were subcutaneously injected into the right flank area of the mice. After the tumours grew to an area of about $100 \mathrm{~mm}^{3}$, the tumour-bearing mice were randomly divided into the control group, Pae group (purity $>98 \%, 100 \mathrm{mg} \mathrm{kg}^{-1}$, Aladdin Ltd.), cisplatin (Cis, purity > $99 \%, 5 \mathrm{mg} \mathrm{kg}^{-1}$, Sigma-Aldrich, group, and Pae $\left(100 \mathrm{mg} \mathrm{kg}^{-1}\right)+\mathrm{Cis}\left(5 \mathrm{mg} \mathrm{kg}^{-1}\right)$ group, with five mice in each group. They were given an intraperitoneal injection daily for 20 days. Body weight and tumour growth were measured every four days. The size of tumours was measured according to the following formula: tumour volume $=\pi / 6 \times L \times W^{2}$, in which $L$ and $W$ represent the major and minor diameter, respectively. At the end of the study, the mice were sacrificed and tumour tissue was weighed prior to immunohistochemistry.

\section{Immunohistochemistry and immunocytochemistry}

RT4 cells were treated with $50 \mu \mathrm{M}$ Pae for $48 \mathrm{~h}$ and fixed in $4 \%$ paraformaldehyde for $20 \mathrm{~min}$ at room temperature. The cells were incubated with anti-STAT3 overnight at $4{ }^{\circ} \mathrm{C}$ 
(1:100, Santa Cruz), followed by incubation with FITC-conjugated secondary antibodies for $1 \mathrm{~h}$ at room temperature. Finally, the cells were labelled with a 4',6-diamidino-2-phenylindole (DAPI) solution and observed with a confocal laser scanning microscope (TCS SP8, Leica Microsystems, Germany). Moreover, tumour tissues were fixed with $10 \%$ formaldehyde for $24 \mathrm{~h}$. Paraffin-embedded slides were conventionally prepared. The sections were deparaffinised in xylene, rehydrated by descending grades of ethanol, and incubated with an antibody against p-STAT3 (Santa Cruz Biotechnology). After washing with PBS, slides were incubated with the biotinylated secondary antibody (Dako). The immunostained cells were quantified by counting the brown cells and the total number of cells in five randomly selected fields at $400 \times$ magnification.

\section{Statistical analysis}

The data are expressed as the mean \pm SD. The statistical difference was determined by one-way ANOVA tests and Student's $t$-test. A $P$ value less than 0.05 was considered statistically significant. All statistical analyses were performed using the SPSS version 13.0 statistical software (SPSS, USA).

\section{RESULTS AND DISCUSSION}

The cytotoxic effect of Pae on BCa cells was evaluated by the MTT assay after exposure to Pae. As shown in Fig. 1b,c, Pae treatment decreased RT4, J82 and 253J cell viability

a)

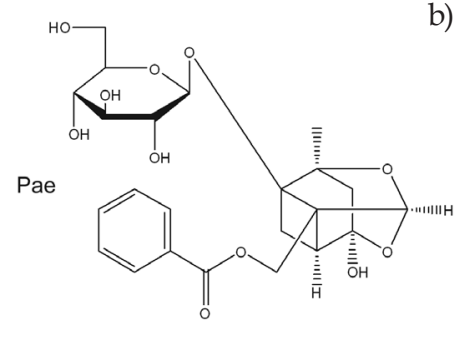

b)

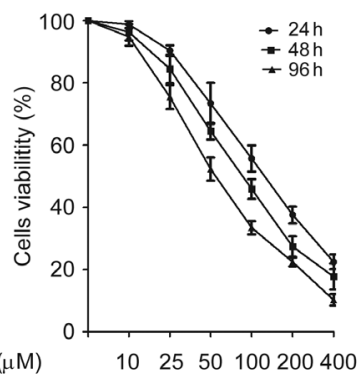

c)

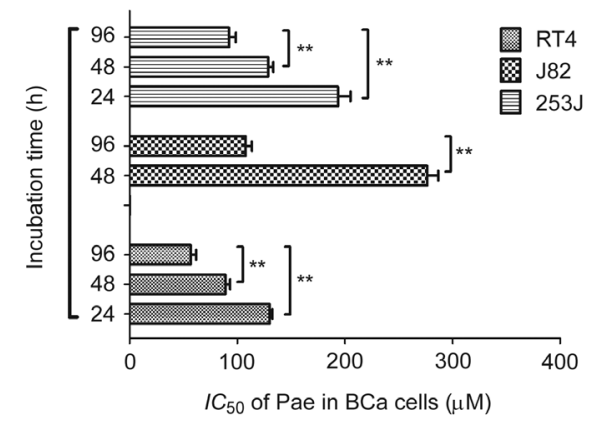

Fig. 1. Cytotoxicity of Pae in BCa cells. a) Pae chemical structure, b) RT4 cells were incubated with different concentrations of Pae for 24, 48 and $96 \mathrm{~h}, \mathrm{c}) I C_{50}$ of Pae. Data are presented as the mean $\pm \mathrm{SD}, n=3{ }^{\mathrm{b}} p<0.01$ compared to the $96 \mathrm{~h}$ group. 
a) a1

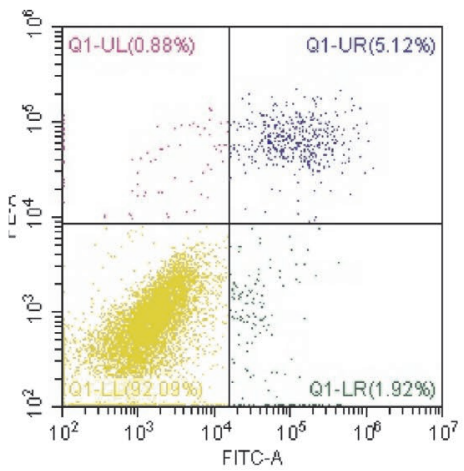

a2

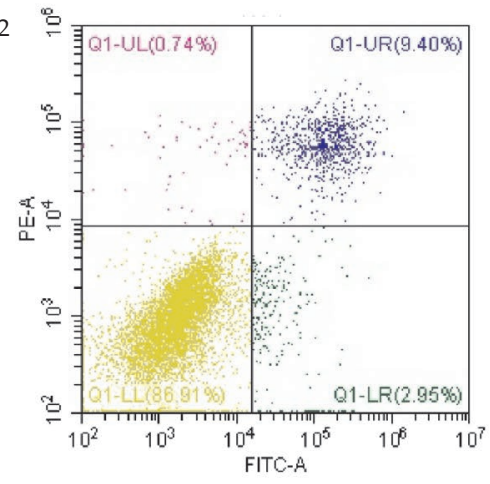

a3

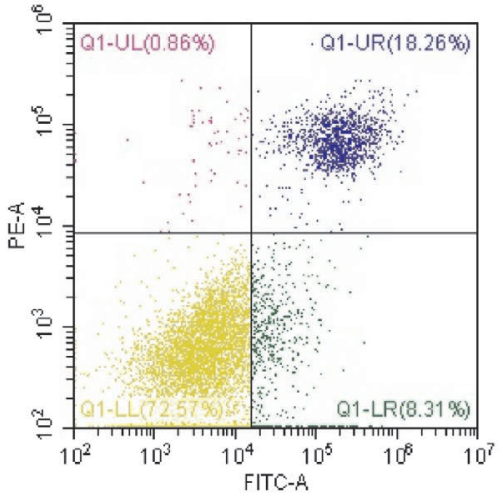

b)

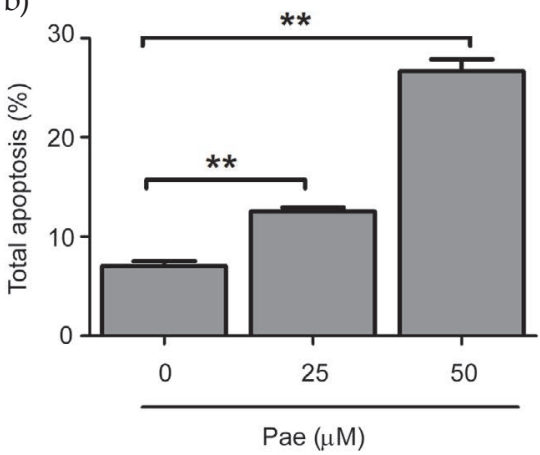

Fig. 2. Effects of Pae on apoptosis of RT4 cells. a) Representative apoptosis data analysed by flow cytometry using an AnnexinV-FITC in RT4 cells are shown for a1 (control group), a2 (Pae $25 \mu \mathrm{M}$ group) and a3 (Pae $50 \mu \mathrm{M}$ group), b) quantitative apoptosis data in Pae treated RT4 cells are shown. Data are presented as the mean $\pm \mathrm{SD}, n=3$; ${ }^{* *} p<0.01$ compared to the control group (without Pae treatment).

in a dose-dependent and time-dependent manner. The $I C_{50}$ values of Pae in the RT4 group were lower than that in J82 and 253J cells $(p<0.01)$. Consequently, RT4 was used for the following study.

We further examined whether Pae could induce apoptosis in RT4 cells. As seen in Fig. 2, Pae treatment induced total apoptosis in RT4 cells. Moreover, we also noticed that Pae-treated RT4 cells showed higher levels of early and late apoptotic rates compared to those of the control group $(p<0.01$, Table I). To determine the mechanism underlying the Pae-mediated apoptosis in RT4 cells, we investigated the effects on caspase3, caspase8 and caspase9 activities. The results showed that Pae treatment increased the activities of caspase3, caspase 8 and caspase9 in RT4 cells ( $p<0.01$, Table II). Moreover, it should be noted that Bax expression increased and Bcl-2 expression decreased in RT4 cells that were exposed to Pae $(p<0.01$, Fig. 3a,b).

As STAT3 plays an important role in BCa cell proliferation and survival, we investigated whether Pae modulates constitutive STAT3 activation in RT4 cells. Fig. 4A-4B show 
Table I. Effects of Pae on early and late apoptosis of RT4 cells

\begin{tabular}{ccc}
\hline Concentration $(\mu \mathrm{M})$ & Early apoptosis $(\%)$ & Late apoptosis $(\%)$ \\
\hline 0 & $1.88 \pm 0.26$ & $5.20 \pm 0.62$ \\
25 & $3.07 \pm 0.34^{* *}$ & $9.50 \pm 0.52^{* *}$ \\
50 & $8.45 \pm 0.41^{* *}$ & $18.20 \pm 1.30^{* *}$ \\
\hline
\end{tabular}

Data are presented as the mean $\pm \mathrm{SD}, n=3 ;^{* *} p<0.01$ compared to the control group (without Pae treatment).

Table II. Effects of Pae on caspase activities of RT4 cells

\begin{tabular}{cccc}
\hline Concentration $(\mu \mathrm{M})$ & Caspase-3 activity $(\mathrm{IU})$ & Caspase- 8 activity $(\mathrm{IU})$ & Caspase-9 activity $(\mathrm{IU})$ \\
\hline 0 & $1.88 \pm 0.26^{\mathrm{a}}$ & $1.51 \pm 0.16$ & $2.20 \pm 0.41$ \\
25 & $4.62 \pm 0.50^{\mathrm{b}}$ & $6.90 \pm 0.75^{\mathrm{b}}$ & $5.08 \pm 0.52^{\mathrm{b}}$ \\
50 & $12.2 \pm 0.92^{\mathrm{b}}$ & $8.56 \pm 0.56^{\mathrm{b}}$ & $11.50 \pm 3.73^{\mathrm{b}}$
\end{tabular}

Data presented as the mean $\pm \mathrm{SD}, n=3 ;{ }^{\mathrm{b}} p<0.01$ compared to the control group (without Pae treatment).

that Pae incubation inhibited STAT3 phosphorylation in RT4 cells $(p<0.01)$. Moreover, in view of the nuclear translocation of STAT3 being central to the biological function, we determined whether Pae suppressed nuclear translocation of STAT3. As shown in Fig. 4c, Pae treatment inhibited the translocation of STAT3 to the nucleus in RT4 cells.

To determine if Pae could inhibit tumour growth in vivo, we studied the effects of Pae on RT4 cell xenografts in nude mice. As shown in Fig. 5B-5C, administration of Pae significantly reduced the weight and volume of tumour tissue $(p<0.01)$. Interestingly, we noticed that Pae and Cis showed synergistically inhibitory effects on the growth of tumour

a)

$$
\text { Bax }
$$

$\mathrm{Bcl} 2$

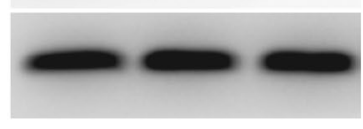

Pae $(\mu \mathrm{M}) \quad 0 \quad 25 \quad 50$ b)

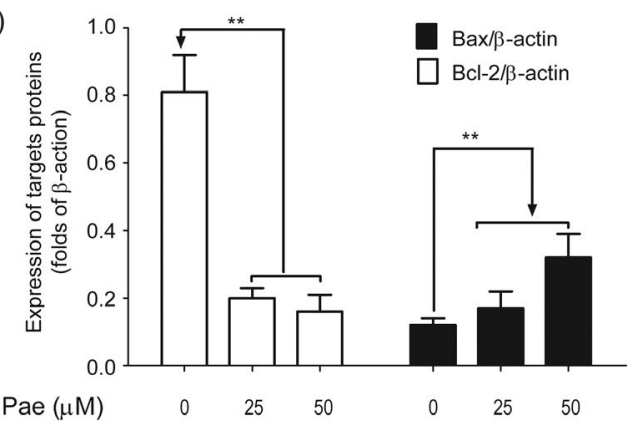

Fig. 3. Effects of Pae on Bax and Bcl-2 levels. a) Representative immunoblotting bands of Bax and Bcl-2. $\beta$-actin was used as an internal reference, $b$ ) quantitative data of the levels of Bax and Bcl-2. Data are presented as the mean $\pm \mathrm{SD}, n=3 ;^{* *} p<0.01$ compared to the control group (without Pae treatment). 
a) Pae $(\mu \mathrm{M})$

0

25

50

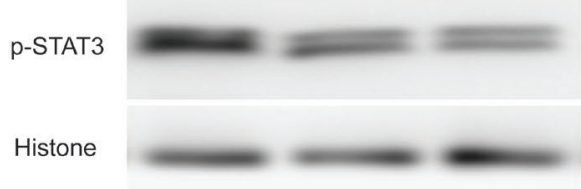

b)

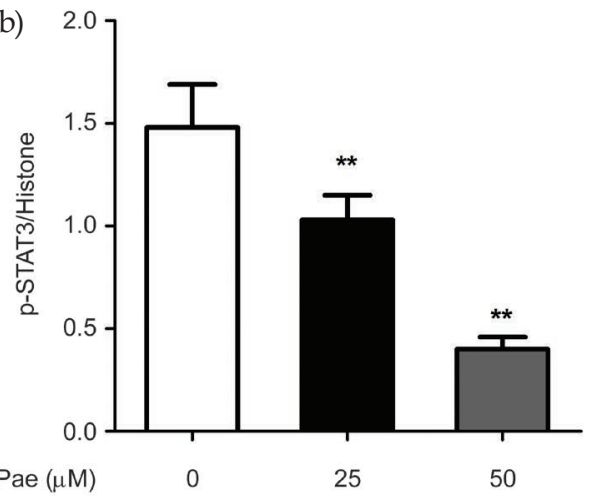

C)

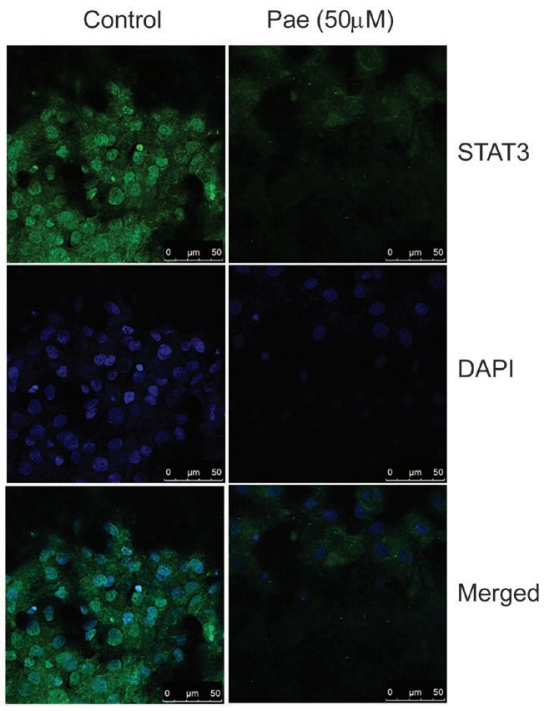

Fig. 4. Effects of Pae on p-STAT3 expression and nuclear translocation in vitro. a) Representative immunoblotting bands of p-STAT3. Histone was used as an internal reference, b) quantitative data of p-STAT3 expression, c) RT4 cells were treated with $50 \mu \mathrm{M}$ Pae for $48 \mathrm{~h}$. Data are presented as the mean $\pm \mathrm{SD}, n=3 ;{ }^{* *} p<0.01$ compared to the control group (without Pae treatment).

tissue $(p<0.01)$. In addition, Pae treatment had no effects on the body mass of tumourbearing mice, while Cis significantly reduced the weight (Fig. $5 \mathrm{a}, p<0.01$ ). Furthermore, the body weight of the combined group was not statistically different from that of the control group and the Pae group at the end of the study. Moreover, in Fig. $5 \mathrm{~d}$, the rates of p-STAT3-positive cells in control or Pae-treated mice were $46.0 \pm 4.5 \%$ and $12.0 \pm 1.3 \%$, respectively $(p<0.01)$, suggesting that Pae significantly suppresses the activation of STAT3 in RT4-bearing mice.

Naturally derived compounds are a prime source of highly effective conventional anti-cancer drugs. Pae, extracted from RPA, is a natural anti-cancer medicine. Many pharmacological studies have reported that Pae exerts anti-cancer and anti-proliferative activity in cultured tumour cells, as well as in tumour xenograft models (12-15). In 2016, Lin et al. (16) reported the anti-proliferative properties of RPA extract on BCa, both in vitro and in vivo. In their research, they also found that the extract showed lower cytotoxicity to normal cells compared to that in BCa cells. It is well known that Pae is the main active ingredient in the RPA extract (17). Therefore, the evidence suggests that Pae may inhibit the growth of BCa tumours. Our investigation first explored the anti-tumour effects of Pae in BCa in both in vitro and in vivo models. Our data demonstrated that Pae effectively inhibits BCa cell (RT4, J82 and 253J) growth in vitro. The $I C_{50}$ of Pae for inhibiting RT4 growth is 88.93 $\mu \mathrm{M}$, which is similar to previous studies conducted in BxPC-3 cells (12). In the in vivo study, we observed that oral administration of Pae significantly inhibited tumour growth and that Pae \& Cis showed synergistically inhibitory effects on the growth of tumours. Some 
a)

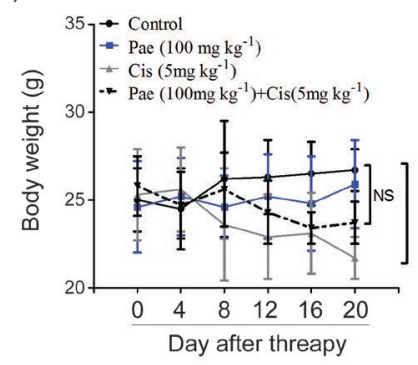

d) $\quad$ d 1

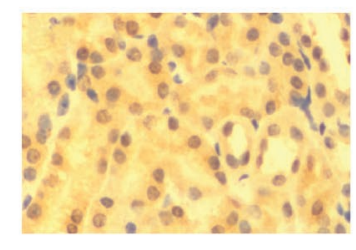

b)

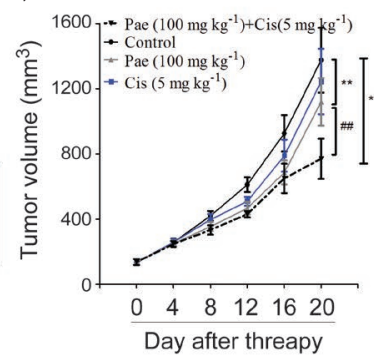

$\mathrm{d} 2$

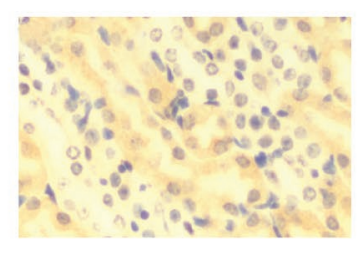

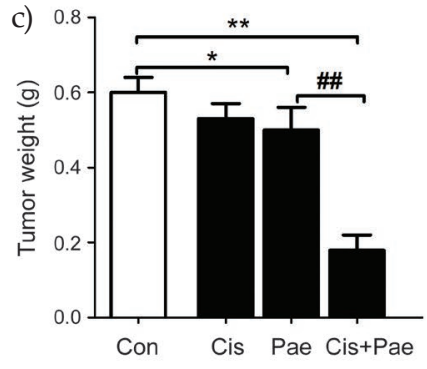

d3

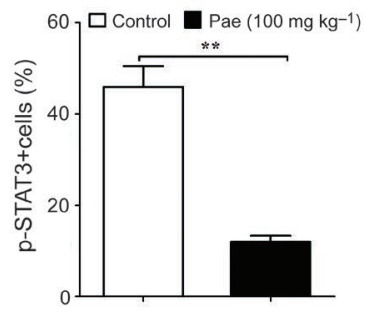

Fig. 5. Effects of Pae on tumour growth in RT4 xenograft mice. a) Body mass, b) tumour volume, c) tumour mass, d) tumour tissues from the control (d1) and Pae group (d2) were stained with pSTAT3 antibody. Representative images were recorded at a magnification of 400x, and quantification of the immunohistochemical assay is presented as the percentage of positively stained cells in $\mathrm{d} 3$. Data are presented as the mean $\pm \mathrm{SD}, n=5 ;{ }^{*} p<0.05,{ }^{* *} p<0.01$ compared to the control group; ${ }^{\# \#} p<0.01 \mathrm{com}$ pared to the Pae group; NS: No significance.

previously published papers have shown similar results. For instance, in 2008, Wu et al. (18) reported that Pae enhanced 5-fluorouracil-induced apoptosis in human gastric carcinoma cells, and Fang et al. (19) concluded that Pae at non-toxic concentrations effectively modulated multidrug resistance of a human gastric cancer cell line via inhibition of nuclear factor-kappa B activation. Similarly, Hao et al. (12) previously confirmed that Pae enhanced the effect of erlotinib in pancreatic cancer cells by directly suppressing human epidermal growth factor receptor-3 activation. Based on these findings, we may speculate that Pae has a synergistic effect with chemotherapeutic drugs regarding the molecular mechanisms or intracellular pharmacokinetic processes. An unexpected finding in the present study was that Pae had no effect on body weight but neutralized body mass loss induced by Cis. These interesting results may be explained as follows: Pae shows high selectivity with an anti-tumour dose and inhibits the growth of tumour cells rather than normal cells. It was shown in a previous study that Pae used against human colon carcinoma in a xenograft tumour did not lead to body weight loss or alterations in food consumption, while docetaxel resulted in a $23.6 \pm 3.1 \%$ mass loss during the same time (20). Moreover, Pae did not show any effects on the numbers of leukocytes, erythrocytes and haemoglobin, while docetaxel significantly reduced those of leukocytes (20), indicating the safety of Pae. Moreover, Pae shows favourable dual immunomodulatory effects and normalizes the function of the immune system in various disease states. For example, Pae was shown to enhance and 
inhibit spleen lymphocyte proliferation at different concentrations (21). Unlike Pae, it is well known that Cis can attack and destroy the growth of tumour cells and immune cells simultaneously. In addition, Pae attenuated Cis-induced body loss in tumour-bearing mice, indicating that Pae reduced chemotherapy -induced toxicity to the immune system. In previous investigations, Pae was shown to decrease myelosuppression induced by chemotherapy (22). We suspect that Pae could increase the defensive power of the immune system in response to chemotherapy. These results indicate that Pae is a potential antitumour adjuvant in the prevention of BCa development.

Apoptosis is a process of programmed cell death. In general, two major apoptotic pathways have been described for mammalian cells. The first pathway, called the extrinsic pathway, is characterized by activation of caspase- 8 . The second pathway, called the intrinsic pathway, is characterized by activation of caspase-9. Activation of caspase- 8 and caspase-9 could trigger activation of the downstream caspase-3. Moreover, the intrinsic pathway is also modulated by Bcl-2 family members, which play key regulatory roles in apoptosis (23-24). In previous studies, Pae induced apoptosis in several types of tumour cells with activation of caspases. For instance, Wang et al. (14) reported that Pae induced apoptosis in HT 29 cells and activated caspase-3 and caspase-8. Wang et al. (25) found that Pae effectively increased cell apoptosis and augmented the activation of caspase- 3 and caspase-9 in SKO-007 cells. In our study, we found that induction of apoptosis was observed in RT4 cells exposed to Pae, followed by activation of caspase-3, caspase- 8 and caspase-9. Furthermore, Pae also increased the level of Bax and simultaneously reduced the level of Bcl-2. These results demonstrated that both the extrinsic and intrinsic pathways seemed to be engaged in the Pae-treated RT4 cell types, which is in agreement with previously published results.

Many studies have been conducted to reveal the mechanisms of the Pae anti-tumour action. Some previous studies reported that Pae exerted its anti-tumour activity via suppressing extracellular regulated protein kinases and phosphatidylinositol 3-kinase/protein kinase B signalling pathways (26-27). Moreover, other studies concluded that Pae induced apoptosis in hepatocellular carcinoma cells by down-regulating prostaglandin E receptor 2 (28). It is accepted that the STAT3 signalling pathway is over-activated and contributes to tumour progression in malignancies. Constitutive activation of STAT3 resulted in cell growth and survival in human solid tumour malignancies and the up-regulation of anti-apoptotic proteins (Bcl-2, Survivin) (29-30). Therefore, deactivation of STAT3 is associated with the induction of apoptosis and conveyance of anti-tumour effects. In 2015, Zheng et al. (15) demonstrated that Pae significantly inhibited total expression of pSTAT3 in MGC-803 cells and that over-expression of STAT3 reversed the effect of Pae on the proliferation of MGC-803. As the tumour-promoting effects are ultimately regulated by the STAT3-dependent transcriptional regulation of downstream oncogenes, we focused on the nuclear level of p-STAT3. Our results showed that Pae reduced the expression of nuclear p-STAT3 and demonstrated that Pae also decreased p-STAT3-positive cells numbers in vivo. As expected, we also found that Pae inhibited translocation of STAT3 to the nucleus in RT4 cells. It is noteworthy that a recent study conducted by Nie et al. (13) found rapid down-regulation of STAT3 that was consistent with its accelerated degradation, and the pro-degradation effect of Pae on STAT3 was mainly through the ubiquitin-proteasome pathway. Therefore, these findings clearly indicate that deactivation of STAT3 by Pae contributes to its inhibitory effects on BCa development. 


\section{CONCLUSIONS}

The present study demonstrated that Pae caused growth inhibition in human BCa cells, both in vivo and in vitro. To the best of our knowledge, our data support the hypothesis that Pae may be an effective anti-tumour agent for the treatment of human BCa. Its action is regulated, at least in part, by deactivation of STAT3 and inhibition of subsequent nuclear translocation.

Acknowledgements. - This work was financially supported by the science and technology project of Ningbo City (No. 2009A610143).

\section{REFERENCES}

1. M. Peng, Y. Huang, T. Tao, C. Y. Peng, Q. Su, W. Xu, K. O. Darko, X. Tao and X. Yang, Metformin and gefitinib cooperate to inhibit bladder cancer growth via both AMPK and EGFR pathways joining at Akt and Erk, Sci. Rep. 6 (2016) 28611; https://doi.org/10.1038/srep28611

2. R. Arantes-Rodrigues, R. Pinto-Leite, L. Fidalgo-Gonçalves, C. Palmeira, L. Santos, A. Colaço and P. Oliveira, Synergistic effect between cisplatin and sunitinib malate on human urinary bladder-cancer cell lines, Biomed. Res. Int. (2013) 791406; https://doi.org/10.1155/2013/791406

3. E. W. Gerharz, A. Månsson and W. Månsson, Quality of life in patients with bladder cancer, Urol. Oncol. 23 (2005) 201-207; https://doi.org/10.1016/j.urolonc.2005.03.005

4. E. J. Hillmer, H. Zhang, H. S. Li and S. S. Watowich, STAT3 signaling in immunity, Cytokine Growth Factor Rev. 31 (2016) 1-15; https://doi.org/10.1016/j.cytogfr.2016.05.001

5. S. Bhattacharya, R. M. Ray and L. R. Johnson, STAT3-mediated transcription of Bcl-2, Mcl-1 and c-IAP2 prevents apoptosis in polyamine-depleted cells, Biochem. J. 392 (2005) 335-344; https://doi. org/ 10.1042/BJ20050465

6. J. Bromberg and J. E. Jr. Darnell, The role of STATs in transcriptional control and their impact on cellular function, Oncogene 19 (2000) 2468-2473; https://doi.org/10.1038/sj.onc.1203476

7. M. Degoricija, M. Situm, J. Korać, A. Miljković, K. Matić, M. Paradžik, I. Marinović Terzić, A. Jerončić, S. Tomić and J. Terzić, High NF-kB and STAT3 activity in human urothelial carcinoma: a pilot study, World. J. Urol. 32 (2014) 1469-1475; https://doi.org/10.1007/s00345-014-1237-1

8. B. Zhang , Z. Lu, Y. Hou, J. Hu and C. Wang, The effects of STAT3 and Survivin silencing on the growth of human bladder carcinoma cells, Tumour Biol. 35 (2014) 5401-5407; https://doi.org/10.1007/ s13277-014- 1704-8

9. H. H. Yeh, R. Giri, T. Y. Chang, C. Y. Chou, W. C. Su and H. S. Liu, Ha-ras oncogene-induced Stat3 phosphorylation enhances oncogenicity of the cell, DNA. Cell. Biol. 28 (2009) 131-139; https://doi. org/10.1089/dna.2008.0762

10. R. J. Chen, Y. S. Ho, H. R. Guo and Y. J. Wang, Long-term nicotine exposure-induced chemoresistance is mediated by activation of Stat 3 and downregulation of ERK1/2 via nAChR and betaadrenoceptors in human bladder cancer cells, Toxicol. Sci. 115 (2010) 118-130; https://doi.org/10.1093/ toxsci/kfq028

11. S. Parker, B. May, C. Zhang, A. L. Zhang, C. Lu and C. C. Xue, A pharmacological review of bioactive constituents of Paeonia lactiflora Pallas and Paeonia veitchii Lynch, Phytother. Res. 30 (2016) 1445-1473; https://doi.org/10.1002/ptr.5653

12. J. Hao, X. Yang, X. L. Ding, L. M. Guo, C. H. Zhu, W. Ji, T. Zhou and X. Z. Wu, Paeoniflorin potentiates the inhibitory effects of erlotinib in pancreatic cancer cell lines by reducing ErbB3 phosphorylation, Sci. Rep. 6 (2016) 32809; https://doi.org/10.1038/srep32809 
13. X. H. Nie, J. Ou-yang, Y. Xing, D. Y. Li, X. Y. Dong, R. E. Liu and R. X. Xu, Paeoniflorin inhibits human glioma cells via STAT3 degradation by the ubiquitin-proteasome pathway, Drug. Des. Devel. Ther. 9 (2015) 5611-5622; https://doi.org/10.2147/DDDT.S93912

14. H. Wang, H. Zhou, C. X. Wang, Y. S. Li, H. Y. Xie, J. D. Luo and Y. Zhou, Paeoniflorin inhibits growth of human colorectal carcinoma HT 29 cells in vitro and in vivo, Food Chem. Toxicol. 50 (2012) 1560-1567; https://doi.org/10.1016/j.fct.2012.01.035

15. Y. B. Zheng, G. C. Xiao, S. L. Tong, Y. Ding, Q. S. Wang, S. B. Li and Z. N. Hao, Paeoniflorin inhibits human gastric carcinoma cell proliferation through up-regulation of microRNA-124 and suppression of PI3K/Akt and STAT3 signaling, World J. Gastroenterol. 21 (2015) 7197-7207; https://doi. org/10.3748/wjg. v21. i23.7197

16. M. Y. Lin, S. Y. Chiang, Y. Z. Li, M. F. Chen, Y. S. Chen, J. Y. Wu and Y. W. Liu, Anti-tumor effect of Radix Paeoniae rubra extract on mice bladder tumors using intravesical therapy, Oncol. Lett. 12 (2016) 904-910; https://doi.org/10.3892/ol.2016.4698

17. J. Liang, F. Xu, Y. Z. Zhang., S. Huang, X. Y. Zang, X. Zhao, L. Zhang, M. Y. Shang, D. H. Yang, X. Wang and S. Q. Cai, The profiling and identification of the absorbed constituents and metabolites of Paeoniae radix rubra decoction in rat plasma and urine by the HPLC-DAD-ESI-IT-TOF-MS(n) technique: a novel strategy for the systematic screening and identification of absorbed constituents and metabolites from traditional Chinese medicines, J. Pharm. Biomed. Anal. 83 (2013) 108-121; https://doi.org/10.1016/j.jpba. 2013.04.029

18. H. Wu, W. Li, T. Wang, Y. Shu and P. Liu, Paeoniflorin suppress NF-kappaB activation through modulation of I kappaB alpha and enhances 5-fluorouracil-induced apoptosis in human gastric carcinoma cells, Biomed. Pharmacother. 62 (2008) 659-666; https://doi.org/10.1016/j.biopha.2008.08.002

19. S. Fang, W. Zhu, Y. Zhang, Y. Shu and P. Liu, Paeoniflorin modulates multidrug resistance of a human gastric cancer cell line via the inhibition of NF-kB activation, Mol. Med. Rep. 5 (2012) 351356; https://doi.org/10.3892/mmr.2011.652

20. H. Wang, H. Zhou, C. X. Wang, Y. S. Li, H. Y. Xie, J. D. Luo and Y. Zhou, Paeoniflorin inhibits growth of human colorectal carcinoma HT 29 cells in vitro and in vivo, Food. Chem. Toxicol. 50 (2012) 1560-1567; https://doi.org/10.1016/j.fct.2012.01.035

21. Z. D. Ge, A. W. Zhou, B. Wang, Y. X. Shen, C. H. Ding, A. P. Zhang, W. Wei and S. Y. Xu, Immunoregulatory effects of total glucosides of Paeony (TGP), Paeoniflorin (PF) and TGP removed PF on adjuvant arthritic rats, Chin. Pharmacol. Bull. 11 (1995) 303-305; https://doi.org/10.3321/j. issn:1001-1978.1995.04.012

22. Y. L. Zhu, L. Y. Wang, J. X. Wang, C. Wang, C. L. Wang, D. P. Zhao, Z. C. Wang and J. J. Zhang, Protective effects of paeoniflorin and albiflorin on chemotherapy-induced myelosuppression in mice, Chin. J. Nat. Med. 14 (2016) 599-606; https://doi.org/10.1016/S1875-5364(16)30070-X

23. S. Goldar, M. S. Khaniani, S. M. Derakhshan and B. Baradaran, Molecular mechanisms of apoptosis and roles in cancer development and treatment, Asian Pac. J. Cancer. Prev. 16 (2015) 2129-2144; https://doi.org/10.7314/APJCP.2015.16.6.2129

24. Z. Jin and W. S. El-Deiry, Overview of cell death signaling pathways, Cancer Biol. Ther. 4 (2005) 139-163; https://doi.org/10.4161/cbt.4.2.1508

25. S. Wang and W. Liu, Paeoniflorin inhibits proliferation and promotes apoptosis of multiple myeloma cells via its effects on microRNA-29b and matrix metalloproteinase-2, Mol. Med. Rep. 14 (2016) 2143-2149; https://doi.org/10.3892/mmr.2016.5498

26. N. Yang, H. Cui, F. Han, L. Zhang, T. Huang, Y. Zhou and J. Zhou, Paeoniflorin inhibits human pancreatic cancer cell apoptosis via suppression of MMP-9 and ERK signaling, Oncol. Lett. 12 (2016) 1471-1476; https://doi.org/10.3892/ol.2016.4761 
27. Z. Zhou, S. Wang, C. Song and Z. Hu, Paeoniflorin prevents hypoxia-induced epithelial-mesenchymal transition in human breast cancer cells, Onco. Targets Ther. 9 (2016) 2511-2518; https://doi. org/10.2147/OTT.S102422

28. S. Hu, W. Sun, W. Wei, D. Wang, J. Jin, J. Wu, J. Chen, H. Wu and Q. Wang, Involvement of the prostaglandin E receptor EP2 in paeoniflorin-induced human hepatoma cell apoptosis, Anticancer Drugs. 24 (2013) 140-149; https://doi.org/10.1097/CAD.0b013e32835a4dac

29. H. Yu and R. Jove, The STATs of cancer--new molecular targets come of age, Nat. Rev. Cancer. 4 (2004) 97-105; https://doi.org/10.1038/nrc1275

30. P. K. Epling-Burnette, J. H. Liu, R. Catlett-Falcone, J. Turkson, M. Oshiro, R. Kothapalli, Y. Li, J. M. Wang, H. F.Yang-Yen, J. Karras, R. Jove and T. P. Jr. Loughran, Inhibition of STAT3 signaling leads to apoptosis of leukemic large granular lymphocytes and decreased Mcl-1 expression, J. Clin. Invest. 107 (2001) 351-362; https://doi.org/10.1172/JCI9940 